\title{
EDITORIAL
}

\section{Emerging Concepts in Geriatric Psychiatry: an Update on the Canadian Academy of Geriatric Psychiatry Annual Scientific Meeting}

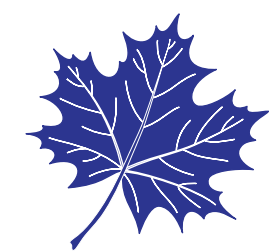

\author{
Zahinoor Ismail, MD, FRCPC, and Corinne Fischer, MD, FRCPC \\ Hotchkiss Brain Institute, University of Calgary, Calgary, AB, Canada; Department of Psychiatry, University of Toronto
}

DOI:http://dx.doi.org/10.5770/cgj.17.121

The Annual Scientific Meeting of the Canadian Academy of Geriatric Psychiatry was held in Ottawa, Ontario on September $25^{\text {th }} 2013$. Given greater weight this year due to the inaugural Royal College Geriatric Psychiatry subspecialty certification exam being written across the country the following day, the meeting proved to be not only a venue for dissemination of current knowledge in Geriatric Psychiatry, but also for networking and meeting with colleagues. Preceded by the $2^{\text {nd }}$ Annual CAGP Review Course, the theme of the ASM, "Emerging Concepts in Geriatric Psychiatry", was all the more relevant in the context of the exam and the changes in Geriatric Psychiatry certification in Canada.

The keynote presentation this year was given by Dr. Benoit Mulsant on the topic of the changes relevant to Geriatric Psychiatry that were introduced with the publication of the $5^{\text {th }}$ edition of the Diagnostic and Statistical Manual of Psychiatric Disorders by the American Psychiatric Association. Dr. Mark Rapoport has written an editorial discussing Dr. Mulsant's presentation with his own reflections, as well. The plenary session was a panel entitled "Advances in Therapeutic Brain Stimulation", presented by Dr. Daniel Blumberger and Dr. Paul Lespérance. Dr. Blumberger started by categorizing brain stimulation into non-convulsive, convulsive, and surgical intervention categories. As an example of non-convulsive treatment, he discussed the advent of Transcranial Direct Current Stimulation, in which low-voltage current is delivered to the prefrontal cortex (PFC) over 20-40 minutes. ${ }^{(1)} \mathrm{He}$ described the mechanism of anode stimulation leading to increased neuronal activity (depolarization), leading to enhanced cortical excitability, leading to the choice of the prefrontal cortex for stimulation. He then discussed electroconvulsive therapy (ECT), and described the importance of ultrabrief pulse width stimuli of $0.3-0.37 \mathrm{~ms}$ and the evidence indicating that it is more effective than bilateral standard pulse width ECT with fewer cognitive side effects. ${ }^{(2)} \mathrm{He}$ also described the use of nortryptiline and lithium for postECT maintenance treatment, and highlighted the need for more research in this area. Dr. Blumberger also described
Magnetic Seizure Therapy ${ }^{(3)}$ as a new advance in brain stimulation. He described it as a high-power Transcranial Magnetic Stimulation (rTMS) device for seizure induction, ideally with less cognitive impairment and faster recovery, compared to ECT, due to more focal electrical fields, narrower pulse widths, and lower anaesthesia requirements. Dr. Lespérance further elaborated on rTMS, looking at the evidence for this treatment modality, and leading a discussion on whether the data is clinically meaningful. (4) He indicated that the parameters of stimulation are still in development and that predictors of response are not yet available. Dr. Lespérance concluded with an update on Vagus Nerve Stimulation, indicating that it is approved for treatment-resistant depression, with sustained remission with no switch to mania and improved cognition compared to ECT. ${ }^{(5)}$ While he described the mechanisms working via deactivation of the orbitofrontal cortex, he acknowledged that there is little data in the geriatric population thus far.

In addition to the keynote and plenary session, this year's annual meeting included a number of additional highlights. These included a workshop on how to disclose a diagnosis of dementia using a person-centred, comprehensive, and progressive approach. Participants were asked to work through disclosure of a diagnosis of dementia using a three-tiered process involving pre-disclosure, disclosure, and post-disclosure. There was also time allocated for reflection. Keeping with the theme of dementia, there was also a workshop devoted to reviewing the $4^{\text {th }}$ Canadian Consensus Conference on the Diagnosis and Treatment of Dementia guidelines ${ }^{(6)}$ with respect to imaging and dementia. Participants had an opportunity to interact directly with members of the expert panel who came up with the guidelines, and increase their knowledge of neuroimaging as it pertains to dementia. In addition, there was a workshop entitled "Too Old for Therapy", in which the principles of Cognitive Behavioural Therapy were adapted for use in older adults.

Further, Dr. Mulsant, in conjunction with Drs. Daniel Blumberger, Zahinoor Ismail, Kiran Rabheru, and Mark Rapoport, led a workshop on developing an algorithmic 
approach to pharmacotherapy of late-life depression (LLD). Participants described their approaches to treatment of LLD at various stages of treatment and tried to integrate this into a stepped-care approach to treatment. ${ }^{(7)}$

Concurrent paper sessions focused on a number of common themes, including use of technology, psychotropic medication, health systems issues, and some controversial topics. There was a session devoted to using an artificial intelligence device in a hospital setting to detect falls in older adults with complex psychiatric issues and then link these events to other factors such as behaviour and medication. Another session provided some practical advice on how to set up a geriatric telepsychiatry service to provide access to patients in remote or rural areas. Dr. Jonathan Crowson from McMaster University explained how he and his allied health team established the Geriatric Psychiatry Telemedicine Network to provide consultation to remote and rural areas in Waterloo and Wellington County. Telemedicine has been used in geriatric psychiatry successfully, although there are some unique challenges. ${ }^{(8)}$ He elaborated on the potential benefits of such a network in terms of improving access to care, and also described some of the barriers, such as establishing a therapeutic alliance with a patient you can only see on camera. A third session focused on adapting a telehealth service to address the needs of residents with behavioural and psychological symptoms of dementia (BPSD) living in remote institutions. Dr. Marie-Andree Bruneau from the University of Montreal discussed how she and her colleagues developed a Telepsychogeriatry (teleformation and teleconsultation) program to connect with two partner institutions in remote and rural Quebec. Research in other sectors of North American, such as the United States, suggests that this may be both clinically useful and cost effective. ${ }^{(9)}$ The purpose of the program was to identify and provide recommendations around the management of patients with BPSD. Factors associated with implementation success and failure were discussed, and the evidence base for teleconsultation in treating BPSD was reviewed. Two sessions focused on psychotropic medication-one addressed concerns about QT prolongation and proposing a risk management tool, and the other examined common medical side effects to psychotropic medication among older adults with mood disorders. In the area of health systems, there was a session focusing on referral patterns among the "oldest old" in the Thunder Bay area and the resource implications of this. Another session looked at a knowledge translation intervention designed to increase the competence of primary care physicians treating patients with dementia. There were two somewhat controversial sessions. The first examined the role of the psychiatrist among terminally ill patients wishing to die in light of new legislation being proposed in Quebec. Dr. Simon Dufour-Turbis from McGill University reviewed the international experience of psychiatrists dealing with physician-assisted suicide/euthanasia. Psychiatrists are frequently called upon to consult in such cases, although their role is not always clear. ${ }^{(10)} \mathrm{He}$ also reviewed the potential challenges associated with the legalization of "medical aid in dying", legislation currently being debated in the Quebec National Assembly. The legislation calls for psychiatrists to be consulted when patient competency is in question. Potential ethical and logistical challenges associated with such assessments were discussed. The session included a review of international experts in this area. The second session postulated that the concept of BPSD may be in decline, as it may lack specificity.

Finally, the CAGP Fellowship Award was given to Dr. Jennifer Brault, who presented her ongoing research into "Exogenous Melatonin for Insomnia in Older Adults-a Meta-analysis". She described the research methodology and her plans for future research. The award for Outstanding Contributions in Geriatric Psychiatry was shared by Drs. Melissa Andrew and Catherine Shea for their devoted and successful efforts over many years to get Geriatric Psychiatry officially recognized at the Royal College level.

In summary, the CAGP ASM was successful in providing current and up-to-date education in Geriatric Psychiatry, with a focus on new approaches and treatments. The 2014 ASM will be held in Toronto on September 9 and 10, in a joint venture with the Canadian Coalition for Seniors Mental Health.

\section{REFERENCES}

1. Berlim MT, Van den Eynde F, Daskalakis ZJ. Clinical utility of transcranial direct current stimulation (tDCS) for treating major depression: a systematic review and meta-analysis of randomized, double-blind and sham-controlled trials. J Psychiat Res. 2013;47(1):1-7.

2. Verwijk Ee, Comijs HC, Kok RM, et al. Neurocognitive effects after brief pulse and ultrabrief pulse unilateral electroconvulsive therapy for major depression: a review. J Affect Disorders. 2012;140(3):233-43.

3. Lisanby SH, Schlaepfer TE, Fisch H-U, et al. Magnetic seizure therapy of major depression. Arch Gen Psychiatry. 2001;58(3):303-05.

4. Rossi S, Hallett M, Rossini PM, et al. Safety, ethical considerations, and application guidelines for the use of transcranial magnetic stimulation in clinical practice and research. Clin Neurophysiol. 2009;120(12):2008-39.

5. Cristancho P, Cristancho MA, Baltuch GH, et al. Effectiveness and safety of vagus nerve stimulation for severe treatment-resistant major depression in clinical practice after FDA approval: outcomes at 1 year. J Clin Psychiatry. 2011;72(10):1376-82.

6. Gauthier S, Patterson C, Chertkow H, et al. Recommendations of the 4th Canadian Consensus Conference on the Diagnosis and Treatment of Dementia (CCCDTD4). Can Geriatr J. 2012;15(4):120-26. 
7. Mulsant BH, Alexopoulos GS, Reynolds CF, et al. Pharmacological treatment of depression in older primary care patients: the PROSPECT algorithm. Int J Geriatr Psychiatry. 2001;16(6):585-92.

8. Jones BN, Ruskin PE. Telemedicine and geriatric psychiatry: directions for future research and policy. J Geriatr Psychiatry Neurol. 2001;14(2):59-62.

9. Lyketsos CG, Roques C, Hovanec L, et al. Telemedicine use and the reduction of psychiatric admissions from a long-term care facility. J Geriatr Psychiatry Neurol. 2001;14(2):76-79.
10. Kelly BD, McLughlin DM. Euthanasia, assisted suicide and psychiatry: a Pandora's box [editorial]. Br J Psychiatry. 2002;181(4):278-79.

Correspondence to: Zahinoor Ismail, MD, FRCPC, Hotchkiss Brain Institute, University of Calgary, 140329 St NW, Calgary, AB T2N 2T9, Canada

E-mail: Zahinoor@gmail.com 